\title{
VIVRE LA PHILOSOPHIE : LES MÉMOIRES COMME EUVRE PHILOSOPHIQUE
}

\author{
Manon Garcia
}

Armand Colin | « Littérature »

2018/3 N 191 | pages 53 à 67

ISSN 0047-4800

ISBN 9782200931872

Article disponible en ligne à l'adresse :

https://www.cairn.info/revue-litterature-2018-3-page-53.htm

Distribution électronique Cairn.info pour Armand Colin.

(C) Armand Colin. Tous droits réservés pour tous pays.

La reproduction ou représentation de cet article, notamment par photocopie, n'est autorisée que dans les limites des conditions générales d'utilisation du site ou, le cas échéant, des conditions générales de la licence souscrite par votre établissement. Toute autre reproduction ou représentation, en tout ou partie, sous quelque forme et de quelque manière que ce soit, est interdite sauf accord préalable et écrit de l'éditeur, en dehors des cas prévus par la législation en vigueur en France. Il est précisé que son stockage dans une base de données est également interdit. 


\section{Vivre la philosophie : les Mémoires comme œuvre philosophique}

En vérité, il n'y a pas de divorce entre la philosophie et la vie ${ }^{l}$.

Simone de Beauvoir

Alors qu'aux États-Unis, en Angleterre, en Allemagne, il est courant de voir sur les tables de philosophie des librairies s'empiler des exemplaires de Pour une morale de l'ambiguité ou du Deuxième Sexe, Simone de Beauvoir est encore loin d'être reconnue comme une philosophe à part entière en France. Au lieu d'apparaître comme une figure importante de la philosophie existentialiste et une pionnière de la philosophie féministe, elle est souvent réduite aux rôles de compagne austère de Sartre, de romancière à succès sans grande originalité ou de figure un peu surannée du féminisme. L'entrée de ses Mémoires dans la Pléiade et le choix de mettre les Mémoires d'une jeune fille rangée au programme de l'agrégation de lettres modernes 2019 témoignent de la reconnaissance grandissante de son œuvre littéraire par le monde universitaire français. Pour autant, la dimension philosophique de son œuvre reste encore largement méconnue en France ${ }^{2}$, ce qui est d'autant plus étonnant que c'est à la philosophe française Michèle Le

1. Simone de Beauvoir, L'Existentialisme et la sagesse des nations, Paris, Nagel, 1948 ; rééd. Gallimard, coll. « Arcades », 2008, p. 9.

2. À ma connaissance, deux ouvrages consacrés à la philosophie beauvoirienne ont été traduits en français : Toril Moi, Simone de Beauvoir. Conflits d'une intellectuelle, 1994, trad. fr. Guillemette Belleteste, Paris, Diderot éditeurs, 1995 et Eva Lundgren-Gothlin, Sexe et existence. La philosophie de Simone de Beauvoir, 1991, trad. fr. Michel Kail et Marie Ploux, Paris, Éditions Michalon, 2001, et le seul ouvrage consacré exclusivement à Beauvoir et publié en France est celui de Michel Kail, Simone de Beauvoir philosophe, Paris, PUF, 2006. Notre ouvrage à paraître intitulé On ne naît pas soumise, on le devient, Paris, Flammarion, 2018, contribuera, je l'espère, au développement des études philosophiques sur Beauvoir en France. 
Dœuff $^{3}$ que l'on doit les premières analyses philosophiques de l'œuvre beauvoirienne.

À cette invisibilité générale s'en ajoute une seconde, spécifique aux Mémoires $^{4}$ : même dans les pays où la recherche philosophique sur Beauvoir se porte au mieux, les philosophes concentrent généralement leurs analyses sur les travaux immédiatement reconnaissables comme philosophiques que sont Pyrrhus et Cinéas, Pour une morale de l'ambiguïté, Le Deuxième Sexe ou encore La Vieillesse et accordent assez peu d'attention aux Mémoires ${ }^{5}$, sinon comme source d'information biographique sur Beauvoir. Deux raisons invitent à remettre en cause cette exclusion : premièrement, prendre au sérieux, contre Beauvoir, l'idée que l'œuvre beauvoirienne est philosophiqueconduit inévitablement à reconnaître, avec Beauvoir, la dimension unifiée de son ouvre. En effet, si ces quarante dernières années, la démonstration a été faite que Beauvoir est une philosophe à part entière, cette démonstration a été menée contre Beauvoir elle-même, qui, en dépit de son parcours universitaire et de ses essais, a toujours refusé ce titre en arguant que seul Sartre était philosophe. Toute étude philosophique de Beauvoir est

3. Michèle Le Dœuff, L'Étude et le Rouet. 1. Des femmes, de la philosophie, etc., Paris, Éditions du Seuil, 1989. En dépit d'un colloque consacré à son œuvre en France en 2016, les travaux de Michèle Le Dœuff sont, comme le soulignent Jean Fornasiero et Margaret Sankey dans l'introduction du numéro de l'Australian Journal of French Studies qui lui est consacré, majoritairement lus, commentés, analysés dans le monde anglophone et, d'une certaine manière, on peut, en dépit de la géographie, faire de Michèle Le Dœuff une des pionnières des études beauvoiriennes en Amérique du Nord (Jean Fornasiero et Margaret Sankey, «Introduction », Australian Journal of French Studies, vol. 40, n 3, 2003, p. 237 243). Sur l'influence de Michèle Le Dœuff sur la jeune génération de spécialistes de Beauvoir dans les années 1980 et 1990, on pourra par exemple se référer au récit que fait Nancy Bauer de sa découverte de Beauvoir à la suite de sa lecture de la traduction anglaise de L'Étude et le Rouet, intitulée Hipparchia's choice, dans Nancy Bauer, «Essai sur Beauvoir, Cavell, etc. », Simone de Beauvoir, dir. Éliane Lecarme-Tabone et Jean-Louis Jeannelle, Paris, L'Herne, coll. «Cahiers de l'Herne », 2012, p. 284-287, ou son commentaire en note de son ouvrage sur Beauvoir : " Il est important pour moi de reconnaître que ce livre, qui offre une méditation brillante et, à mon avis, largement sous-estimée, sur les femmes et la philosophie, dont plus de la moitié est consacrée à Beauvoir et au Deuxième Sexe, constitue la meilleure discussion (ou peut-être devrais-je dire démonstration) que je connaisse de la difficulté d'honorer à la fois les exigences d'un engagement féministe et d'un engagement philosophique. Les réussites de Le Dœuff sont d'autant plus impressionnantes au regard du rejet quasi complet de Beauvoir par les philosophes féministes françaises les plus importantes ces trente dernières années ; je trouve son hérö̈sme et son originalité dans ces circonstances presque incroyables. » (Nancy Bauer, Simone de Beauvoir, Philosophy, and Feminism, New York, Columbia University Press, 2001 p. 243-244).

4. J'adopte ici la convention typographique proposée par Jean-Louis Jeannelle, consistant à utiliser la majuscule pour se référer au genre littéraire des Mémoires. À ce sujet, voir JeanLouis Jeannelle, Écrire ses Mémoires au XX $X^{e}$ siècle : déclin et renouveau, Paris, Gallimard, coll. « Bibliothèque des idées », 2008. Sauf mention contraire, nous utiliserons indifféremment ici le terme d'autobiographie et celui de Mémoires pour qualifier l'œuvre beauvoirienne tant cette œuvre est à la fois autobiographique et mémoriale.

5. Les deux ouvrages importants qui abordent cette question sont Jo-Ann Pilardi, Simone de Beauvoir Writing the Self. Philosophy Becomes Autobiography, Westport, CT Greenwood 
donc fondée sur l'hypothèse que Beauvoir aurait tendance à sous-estimer, voire à masquer son travail de philosophe ${ }^{6}$. Or Beauvoir affirme très explicitement que son travail de mémorialiste est indissociable de son travail de romancière et d'essayiste. Par conséquent, considérer l'œuvre beauvoirienne comme philosophique implique de prendre au sérieux la place qu'occupent les Mémoires dans cet édifice. Un second élément invite à lire les Mémoires de manière philosophique : le lien très fort que la philosophie existentialiste pose entre la philosophie et la vie. Beauvoir y insiste à de nombreuses occasions : l'existentialisme repose sur la conviction que philosophie et vie sont indissociablement liées; la première doit parler de la seconde dans ce que celle-ci a de concret et de pratique, la seconde manifester la liberté fondamentale de tous les êtres humains qu'analyse la première.

Il ne peut être question en un article de rendre compte de la richesse philosophique que contiennent les plus de deux mille pages écrites par Beauvoir dans son cycle. Je montrerai néanmoins que les Mémoires ne sont pas seulement une source d'information biographique permettant d'éclairer la lecture des textes plus clairement théoriques de Beauvoir, mais constituent à proprement parler l'un des éléments de son œuvre philosophique. Pour ce faire, après avoir souligné l'intérêt documentaire des Mémoires, je montrerai comment la tâche d'écrire sa vie provient d'une conception de la philosophie comme « attitude » et comme façon de vivre, qui nécessite d'illustrer des principes abstraits en renvoyant à l'épaisseur et à la complexité d'une vie singulière.

Le premier usage, évident, des Mémoires pour saisir la pensée beauvoirienne est documentaire. Pour qui s'intéresse aussi bien à Beauvoir romancière qu'à Beauvoir philosophe, les Mémoires fournissent des indications biographiques et des remarques de l'écrivaine sur son travail très utiles pour enrichir la lecture du reste de son œuvre. Les informations remplissent plus particulièrement une triple fonction : elles fournissent des éléments biographiques importants pour élucider les influences intellectuelles de Beauvoir, elles prémunissent contre une lecture fautive de ses textes et elles permettent même de lire Beauvoir contre elle-même. En effet, la mémorialiste fournit,

\footnotetext{
6. Dans un des premiers articles ayant joué un role important pour la réception américaine de l'œuvre philosophique de Beauvoir, Margaret Simons cite le passage d'un entretien qu'elle eut avec Beauvoir en mars 1979, dans lequel celle-ci déclarait : "For me a philosopher is someone like Spinoza, Hegel, or like Sartre, someone who builds a grand system and not simply someone who loves philosophy, who can teach it, who can understand it and who can make use of it in essays. [...] Sartre is a philosopher, and I am not, and I never really wanted to be a philosopher. I love philosophy very much, but my work has not been in philosophy. I have created a literary work. My interest has been with novels, memories, and essays like The Second Sex. But that is not philosophy. On the level of philosophy, I have been influenced by Sartre. Obviously, I could not have influenced him, since I did not do philosophy. I critiqued it; I discussed many of his ideas with him, but I had no philosophical influence on Sartre. » (Margaret Simons, « Beauvoir and Sartre: The Philosophical Relationship », Yale French Studies, vol. 72, 1986, p. 168).
} 
directement ou indirectement, des indications précises sur ses lectures et permet ainsi de comprendre son parcours intellectuel ou ses emprunts philosophiques. Ainsi que le montre Nancy Bauer ${ }^{7}$, Beauvoir, comme la majorité des philosophes, s'approprie des philosophèmes hérités de différents auteurs et de différents systèmes : contrairement à ce que son surnom de «Grande Sartreuse » pourrait laisser entendre, elle n'emprunte pas uniquement et pas majoritairement à la philosophie sartrienne, mais s'approprie plutôt, dans une synthèse originale, des approches tirées aussi bien de Hegel que de Husserl, de Marx, de Heidegger, de Bergson, ou de Merleau-Ponty. La lecture des Mémoires met en évidence la diversité de ces influences : par exemple La Force de l'âge en ce qui concerne l'influence progressive de Heidegger sur la pensée de Beauvoir et le rôle que celui-ci aura dans son rejet de la philosophie systématique. Alors qu'en 1931, Beauvoir ignore tout de Heidegger ${ }^{8}$, il est clair qu'elle prend connaissance de la philosophie heideggérienne vers la fin des années 1930. Ses hésitations à propos du système hégélien, dans lequel elle se plonge à l'été 1940, sont mises, en effet, au compte de l'influence de Heidegger :

Plus j'allai, plus - sans cesser de l'admirer - je me séparai d'Hegel. Je savais à présent que, jusque dans la moelle de mes os, j'étais liée à mes contemporains ; je découvris l'envers de cette dépendance : ma responsabilité. Heidegger m'avait convaincue qu'en chaque existant s'accomplit et s'exprime « la réalité humaine»: inversement, chacun l'engage et la compromet tout entière ; selon qu'une société se projette vers la liberté ou s'accommode d'un inerte esclavage, l'individu se saisit comme un homme parmi les hommes, ou comme une fourmi dans une fourmilière : mais nous avons tous le pouvoir de mettre en question le choix collectif, de le récuser ou de l'entériner. J'éprouvai quotidiennement cette équivoque solidarité. Dans cette France occupée, il suffisait de respirer pour consentir à l'oppression ; le suicide même ne m'en aurait pas délivrée, il eût consacré ma défaite ; mon salut se confondait avec celui du pays tout entier. Mais cette situation qui m'était imposée, mes remords m'avaient découvert que j'avais contribué à la créer. L'individu ne se résorbe pas dans l'univers qui l'investit : tout en le supportant il agit sur lui, fût-ce par son immobilité même? .

Ce passage montre de quelle manière son adhésion à l'ontologie heideggérienne la conduira à penser d'une façon très particulière la question de la soumission, du choix et de la responsabilité, en refusant à la fois une approche purement individualiste et une approche structurelle et impersonnelle.

7. Nancy Bauer, Simone de Beauvoir, Philosophy, and Feminism, op. cit.

56 8. Elle raconte qu'en 1931, Sartre publie un texte dans la revue Bifur, et ajoute: «Dans le même numéro parut la traduction de Was ist Metaphysik d'Heidegger : nous n'en vîmes pas l'intérêt car nous n'y comprîmes rien » (Simone de Beauvoir, La Force de l'âge, éd. Jean-Louis Jeannelle, Hélène Baty-Delalande et al., dans Mémoires, t. I, Bibliothèque de la Pléiade, 2018, 
L'importance ici accordée à Heidegger n'est toutefois pas un simple élément biographique : elle conduit le lecteur à ne pas réduire Beauvoir à une disciple de Sartre. Elle permet également de comprendre le rôle que celle-ci attribue à l'existentialisme de Kierkegaard et de Heidegger dans son rejet de la philosophie systématique et, plus largement, dans le changement philosophique radical qui s'opère chez elle à cette période. Car la guerre met fin à l'idéalisme et au détachement que Beauvoir décrit dans La Force de l'âge et la convainc de prendre en compte la situation des individus. Par conséquent, l'accent mis sur Heidegger montre, contrairement à ce que l'on pense souvent, la profondeur des désaccords philosophiques entre Beauvoir et Sartre. Ainsi que Heidegger le soulignera violemment dans sa Lettre sur l'humanisme, l'ontologie qu'il propose dans Etre et Temps a pour ambition de récuser la perspective individualiste qui est celle de Husserl et que Sartre reprendra à son compte, en insistant sur le caractère déterminant de la facticité et de la situation. Beauvoir propose dans La Force de l'âge une chronologie du désaccord profond qui l'oppose à Sartre sur le concept de situation. Première étape : la discussion avec Sartre en 1941 au cours de laquelle elle récuse l'idée que la situation serait une pure facticité à laquelle l'individu libre doit s'arracher et dont il est responsable quelle que soit la situation dans laquelle il se trouve. Beauvoir avance l'exemple d'une femme enfermée dans un harem pour souligner l'absurdité de la thèse de Sartre : selon lui, celle-ci pourrait transcender la facticité au même titre que n'importe quel autre sujet ${ }^{10}$. Si, lors de cette discussion, elle cède de mauvaise grâce, elle réitère sa position en 1944 dans son premier texte philosophique, Pyrrhus et Cinéas ${ }^{11}$ Rétrospectivement, elle présente cet essai comme à la fois en accord avec la conception sartrienne de la liberté et comme une défense, contre Sartre, de l'idée que les possibilités concrètes ne sont pas les mêmes pour tous :

Ainsi, j'essayai de concilier avec les idées de Sartre la tendance que, dans de longues discussions, $\mathrm{j}$ 'avais soutenue contre lui : je rétablissais une hiérarchie entre les situations ; subjectivement, le salut était en tout cas possible ; on n'en devait pas moins préférer le savoir à l'ignorance, la santé à la maladie, la prospérité à la pénurie.

10. Beauvoir relate ainsi son opposition à la position que prendra Sartre dans le chapitre de L'Être et le Néant intitulé « Liberté et facticité : la situation » et qu'il esquisse dès 1941 en captivité : «Les jours suivants, nous discutâmes certains problèmes particuliers et surtout le rapport de la situation et de la liberté. Je soutenais que, du point de vue de la liberté, telle que Sartre la définissait - non pas résignation stoïcienne mais dépassement actif du donné - les situations ne sont pas équivalentes : quel dépassement est possible à la femme enfermée dans un harem ? Même cette claustration, il y a différentes manières de la vivre, me disait Sartre. Je m'obstinai longtemps et je ne cédai que du bout des lèvres. Au fond, j'avais raison. Mais pour défendre ma position, il m'aurait fallu abandonner le terrain de la morale individualiste, donc idéaliste, sur lequel nous nous placions. » (Ibid., p. 759).

11. Id., Pyrrhus et Cinéas (1944), dans Pour une morale de l'ambiguïté, Paris, Gallimard, 1947, coll. « Folio », 2003. 
Je ne désapprouve pas mon souci de fournir à la morale existentialiste un contenu matériel ; l'ennui c'est qu'au moment où je croyais m'évader de l'individualisme, j'y restais enlisée. L'individu ne reçoit une dimension humaine que par la reconnaissance d'autrui, pensais-je ; néanmoins, dans mon essai, la coexistence apparaît comme une espèce d'accident que devrait surmonter chaque existant ; celui-ci commencerait par forger solitairement son projet et demanderait ensuite à la collectivité de le valider : en vérité la société m'investit dès ma naissance ; c'est en son sein et dans ma liaison avec elle que je décide de moi $^{12}$.

À la fin de La Force de l'âge, Beauvoir fait de la guerre - et plus particulièrement la guerre en tant qu'elle était pour elle l'expérience d'une inextinguible présence de la mort - l'occasion d'une sorte de conversion ontologique, inséparable d'un engagement politique dans la société : à l'individualisme attentiste qui était le sien dans les années 1930 se substitue une compréhension de l'individu en tant que situé ${ }^{13}$. Cette conversion apparaît très clairement dans Pour une morale de l'ambiguïté, le deuxième texte philosophique qu'elle publie après-guerre. Celui-ci est émaillé de références plus ou moins explicites à Heidegger et est l'occasion de revenir sur la querelle avec Sartre au sujet de la facticité, toujours à travers l'exemple de la femme enfermée dans le harem ${ }^{14}$. Selon Beauvoir, au contraire de son compagnon dans L'Être et le Néant, dire que les individus sont situés, c'est reconnaître qu'ils sont dans le monde, au sens où ils n'existent pas en dehors du monde, qu'ils façonnent en même temps par leur présence même. La lecture des Mémoires permet ici de comprendre de quelle manière, contre Sartre et grâce à Heidegger, Beauvoir construit progressivement une position philosophique originale, dont la version achevée apparaît dans Le Deuxième Sexe et qui consiste à tenir ensemble une conception existentialiste de l'individu et l'importance des déterminations économiques et sociales. La philosophe n'y apparaît plus comme simple disciple de Sartre. Qui plus est, la mise en évidence d'une telle position va à l'encontre de ce que la mémorialiste écrit elle-même lorsqu'elle récuse toute dimension philosophique de son œuvre. À ce titre, son œuvre mémoriale permet de prendre au sérieux la pensée beauvoirienne contre son auteure même et la tendance au dessaisissement de soi que celle-ci identifie dans Le Deuxième Sexe mieux chez les autres femmes que dans ses propres Mémoires ${ }^{15}$.

12. Id., La Force de l'âge, op. cit., p. 867.

13. « Ma vie cessa d'être un jeu, je connus mes racines, je ne feignis plus d'échapper à ma situation : je tentais de l'assumer. Désormais, la réalité pesa son poids. Par instants, il me semblait odieux de m'en accommoder. » (Ibid., p. 915).

14. Beauvoir y prend fermement, quoique de manière implicite, position contre la conception 58 asociale de la liberté que Sartre développe dans L'Être et le Néant. Elle écrit ainsi : «l'esclave noir du XVIII ${ }^{\mathrm{e}}$ siècle, la musulmane enfermée au fond d'un harem, n'ont aucun instrument qui leur permette d'attaquer, fût-ce en pensée, fût-ce par l'étonnement ou la colère, la civilisation qui les opprime» (id., Pour une morale de l'ambiguïté, op. cit., p. 51).

LITTÉRATURE 15. Sur la façon dont Beauvoir s'attache à masquer dans les Mémoires non seulement tout ce que son œuvre pouvait avoir de philosophique mais aussi toute l'influence que sa pensée a pu 
S'il ne fait aucun doute que les Mémoires présentent un tel intérêt documentaire pour qui s'intéresse à la dimension philosophique de l'œuvre beauvoirienne, ils méritent néanmoins d'être considérés pour ce qu'ils sont, à savoir une œuvre philosophique à part entière. Cette écriture d'une existence singulière et socialement, historiquement, économiquement située vient répondre à certains défis théoriques propres à l'existentialisme et constitue peut-être la meilleure preuve de la thèse de la précédence de l'existence sur l'essence. En effet, le slogan existentialiste selon lequel l'existence précède l'essence ${ }^{16}$ signifie que, contrairement à ce que la philosophie spéculative tient pour vrai depuis Aristote, l'être humain ne peut pas d'abord être défini dans son essence. L'être humain est fondamentalement libre et est, par conséquent, en mesure d'inventer librement son essence par l'existence qu'il mène. À ce titre, la vie des individus dit davantage de leur humanité et de ce qu'ils sont que ce que la philosophie spéculative pourrait dire de leur essence.

Alors que Sartre évoque rarement les conséquences d'une telle thèse sur les liens de la philosophie et de la vie, Beauvoir est, avant même son tournant existentialiste, occupée par cette question ${ }^{17}$ et trouve dans l'existentialisme le moyen de rendre évident ce lien de la vie et de la philosophie qui occupait la diariste dès ses Cahiers de jeunesse. Aux lendemains de la seconde guerre mondiale, Beauvoir publie dans Les Temps modernes plusieurs essais en défense de ce qu'elle s'était résolue, après quelques hésitations ${ }^{18}$, à appeler la philosophie existentialiste. Elle s'y attaque au préjugé selon lequel l'existentialisme serait une philosophie désespérante et réfute en particulier l'idée selon laquelle la pensée spéculative «n'aide pas à vivre et même elle en détourne ${ }^{19}$ » : au contraire, « il n’y a pas de divorce entre la philosophie et la vie. Toute démarche vivante est un choix philosophique et l'ambition d'une philosophie digne de ce nom, c'est d'être un mode de

avoir sur la philosophie sartrienne, on se référera avec profit aux analyses passionnantes de Margaret Simons dans « Beauvoir, Philosophy, and Autobiography », A Companion to Simone de Beauvoir, dir. Laura Hengehold et Nancy Bauer, John Wiley \& Sons, 2017, p. 391-405.

16. Dans la conférence qu'il donne sur l'existentialisme, Sartre fait de cette idée la thèse fondamentale de la philosophie existentialiste (voir Jean-Paul Sartre, L'Existentialisme est un humanisme, Paris, Nagel, 1946).

17. « Nous étions deux mystiques. Sartre avait une foi inconditionnée dans la Beauté qu'il ne séparait pas de l'Art, et moi je donnais à la Vie une valeur suprême. » (Simone de Beauvoir, $L a$ Force de l'âge, op. cit., p. 373).

18. «Roman sur la résistance, il fut aussitôt catalogué roman existentialiste. Ce mot désormais était automatiquement accolé aux œuvres de Sartre et aux miennes. [...] Sartre avait refusé que Gabriel Marcel lui appliquât cette étiquette : "Ma philosophie est une philosophie de l'existence ; l'existentialisme, je ne sais pas ce que c'est." Je partageais son agacement. J'avais écrit mes romans avant même de connaître ce terme, en m'inspirant de mon existence et non pas d'un système. Mais nous protestâmes en vain. Nous finîmes par reprendre à notre compte l'épithète dont tout le monde usait pour nous désigner. » (Id., La Force des choses I, éd. Jean-Louis Jeannelle, dans Mémoires, t. I, Bibliothèque de la Pléiade, 2018, p. 977). 19. Id., L'Existentialisme et la sagesse des nations, op. cit., p. 7. 
vie qui apporte avec soi sa justification ${ }^{20}$. » Cette prise de position fait en filigrane apparaître certaines des thèses majeures de l'existentialisme : s'il n'y a pas de divorce entre la vie et la philosophie, c'est que la philosophie dont il est question prend ses distances avec les systèmes abstraits pour être au plus près de la vie dans sa concrétude, que l'homme ne peut échapper à sa liberté (définie par le fait que l'existence en précède l'essence), et qu'il ne peut y avoir de principes moraux absolus autres que la défense de la liberté. L'existentialisme n'est pas tant une doctrine philosophique, spéculative et abstraite, qu'une « attitude philosophique ${ }^{21}$ » qui donne sens à la vie et reçoit son sens de la vie.

La dimension philosophique des Mémoires est la conséquence de ce lien étroit et à double sens entre la philosophie et la vie. Le cycle entrepris à partir de 1958 est une œuvre philosophique car la vie qui y est dépeinte est conçue par Beauvoir comme telle. Le lien que celle-ci opère entre sa vie et la philosophie se manifeste en particulier par la place qu'elle accorde au bonheur. À la dernière page de Tout compte fait, Beauvoir donne à la communication du « goût de [s] a propre vie ${ }^{22}$ » l'ambition profonde de toute son entreprise mémoriale. En effet, des premières pages des Mémoires d'une jeune fille rangée où il est question de « vitalité fougueuse », de « plénitude » et de «béatitude $»^{23}$, jusqu' aux pages de Tout compte fait dans lesquelles elle identifie ce qui lui a permis non seulement d' " avoir une vie heureuse, mais [d']être heureuse de la vie [qu'elle avait] ${ }^{24}$ », décrire le bonheur qu'elle prend à vivre apparaît comme la tâche fondamentale de son récit. Cette place privilégiée accordée au bonheur manifeste la dimension philosophique de la vie à deux niveaux.

Premièrement, Beauvoir accorde un rôle épistémique fort au bonheur : à plusieurs reprises dans les Mémoires, elle met son goût du bonheur à l'origine de sa quête de la vérité. Cela ne signifie pas simplement que le bonheur a une fonction opératoire ; non seulement il est à l'origine de son goût pour la connaissance, mais il informe ce que cette connaissance doit être. Beauvoir écrit ainsi dans La Force de l'âge :

Le bonheur est une vocation moins commune qu'on imagine. [...] Dans toute mon existence, je n'ai rencontré personne qui fût aussi doué que moi pour le bonheur, personne non plus qui s'y acharnât avec tant d'opiniâtreté. Dès que je l'eus touché, il devint mon unique affaire. [...] Il n'était pas seulement cette effervescence dans mon cœur : il me livrait, pensais-je, la vérité de mon existence et du monde ${ }^{25}$.

20. Ibid., p. 9 .

21. Ibid., p. 27. 22. Id., Tout compte fait, éd. Hélène Baty-Delalande, dans Mémoires, t. II, Bibliothèque de la
Pléiade, 2018, p. 972.

23. Id., Mémoires d'une jeune fille rangée, éd. Éliane Lecarme-Tabone, dans Mémoires, t. I, Bibliothèque de la Pléiade, 2018, p. 9.

LITTÉRATURE

24. Id., Tout compte fait, op. cit., p. 516. 
Contrairement à une conception du bonheur comme éventuel résultat de la quête de la vérité, Beauvoir lui confère une portée épistémique : c'est son goût du bonheur qui la conduit à arpenter le monde d'une manière systématique et exigeante comme en témoignent ses récits de voyage ; c'est ce goût du bonheur qui lui fait mettre en doute le type de connaissance abstraite que propose la philosophie spéculative. Une telle effervescence, qui la fait entrer en transe face à la réalité, lui fait faire une expérience presque physique de la nécessité d'une connaissance du monde n'en niant ni sa présence, ni sa contingence, ni les émotions que celui-ci fait naître ${ }^{26}$. La vérité du monde est indissociable de la subjectivité qui appréhende ce monde et y vit. Vie et philosophie, entendues comme entreprise de connaissance du monde, sont indissociables.

La place centrale accordée au bonheur manifeste également le lien essentiel entre la vie et la philosophie comprise comme recherche de la vie bonne. L'une des thèses majeures de ce que Beauvoir appellera dans Le Deuxième Sexe la «morale existentialiste » consiste à dire que la liberté humaine rend impossible de mettre au point des règles morales fixes et applicables à tous. Au contraire, prendre au sérieux le caractère absolu de cette liberté implique de substituer aux normes morales un idéal de sagesse, qui n'est pas fait de préceptes mais d'un engagement moral quotidien et concret. Beauvoir fait référence à cette question dans La Force de l'âge :

Nous récusions, moi par un antique goût de l'absolu, Sartre par dégoût de l'universel, non seulement les préceptes en cours dans notre société, mais n'importe quelle maxime prétendant s'imposer à tous. Devoir et vertu impliquent l'asservissement de l'individu à des lois extérieures à lui : nous les niions ; à des notions vaines, nous opposions une vérité vivante : la sagesse. Le sage, en effet, établit entre soi et l'univers un équilibre singulier et totalitaire ; la sagesse est indivise, elle ne se laisse pas débiter en tranches, elle ne s'obtient pas par une patiente accumulation de mérites [...] La liberté était notre unique règle. Nous défendions qu'on s'aliénât à des rôles, à des droits, à de complaisantes représentations de $\operatorname{soi}^{27}$.

26. «Je me perdais dans mes admirations, mes joies : "voilà le Castor qui entre en transe !" disait Sartre ; lui, il gardait son sang-froid et il essayait de traduire verbalement ce qu'il voyait. Un après-midi, nous regardions des hauteurs de Saint-Cloud un grand paysage d'arbres et d'eau ; je m'exaltai et je reprochai à Sartre son indifférence : il parlait du fleuve et des forêts beaucoup mieux que moi, mais il ne ressentait rien. [...] Plusieurs fois il m'expliqua qu'un écrivain ne pouvait pas avoir d'autre attitude ; quiconque n'éprouve rien est incapable d'écrire ; mais si la joie, l'horreur nous suffoquent sans que nous les dominions, nous ne saurons pas non plus les exprimer. Parfois je lui donnais raison ; mais, parfois, je me disais que les mots ne retiennent la réalité qu'après l'avoir assassinée ; ils laissent échapper ce qu'il y a en elle de plus important : sa présence. [...] Sartre trouvait oiseux de déplorer cet écart entre le mot et la chose, entre l'œuvre créée et le monde donné : il y voyait au contraire la condition même de la littérature et sa raison d'être ; l'écrivain doit en jouer, et non rêver de l'abolir : ses réussites sont dans cet échec assumé. Soit ; je m'accommodais tout de même difficilement de ce divorce, je voulais faire des livres, mais non renoncer à mes "transes" : j'étais tiraillée. » (Ibid., p. 386-387). 
Le tournant de la guerre conduit Beauvoir à critiquer cette foi illimitée en la liberté, aveugle aux déterminations sociales et économiques. Elle n'en demeure pas moins convaincue que la morale ne peut être faite de préceptes abstraits s'imposant à tous. Vivre en accord avec la morale existentialiste implique non pas de suivre quelques prétextes mais de trouver, grâce à sa liberté, un équilibre entre le monde et soi. Mais s'il n'y existe pas de préceptes assurant que la vie choisie est une vie morale, comment savoir ce qu'est une vie morale ? Une telle révélation ne peut se faire qu'à partir d'exemples singuliers. L'autobiographie entend donc précisément répondre à cette question par l'intermédiaire d'une expérience propre : il s'agit pour Beauvoir de raconter $s a$ vie. Ainsi donne-t-elle non seulement un exemple de vie marquée par la recherche de la sagesse, mais récuse-telle implicitement de plus, en insistant sur le bonheur qui marque sa vie, l'idée que l'existentialisme offre « à l'homme une image de lui-même et de sa condition propre à le désespérer ${ }^{28} »$. Bien au contraire, le bonheur qui traverse le récit de sa vie montre l'existence comme exercice de la liberté et pratique de la sagesse plutôt que comme soumission à des principes moraux abstraits et fait apparaitre l'existentialisme comme une philosophie tournée vers la recherche authentique du bonheur.

Si la première dimension théorique des Mémoires est liée à la teneur essentiellement philosophique de la vie elle-même, la seconde dimension tient également à ce qu'il y a de philosophique dans la vie en tant que celle-ci est racontée dans une œuvre littéraire. On l'a vu au sujet de la morale existentialiste, l'une des tensions de cette philosophie résulte de son apparente contradiction entre une ambition proprement philosophique, donc nécessairement abstraite, et, dans le champ de la philosophie morale, normative, un refus de l'abstraction et de la normativité propres à la philosophie spéculative, idéaliste et universalisante. La thèse sartrienne selon laquelle l'existence précède l'essence en est une parfaite illustration tant son ambition concrète est contredite par l'abstraction de sa formulation. Or une façon de surmonter ce paradoxe est d'abandonner la forme philosophique, nécessairement abstraite et nécessairement universalisante, au profit d'une forme qui fasse émerger l'existence concrète des individus et de leur liberté, ce que la philosophie ne permet pas. Chez Beauvoir, dès L'Invitée, il est clair que la forme romanesque répond au défi de faire apparaître les libertés et les relations humaines dans leur dimension concrète. Pour autant, Beauvoir montre dans ses Mémoires que la fiction romanesque échoue, elle aussi, à saisir la vie dans sa contingence :

J'avais raconté cette période dans Les Mandarins : c'est, pensais-je, en projetant une expérience dans l'imaginaire qu'on en dégage le plus évidemment la signification. Mais je regrettais que le roman échouât toujours à en rendre la 
contingence : les imitations qu'il peut en offrir sont toujours ressaisies par la nécessité. Dans une autobiographie, au contraire, les événements se présentent dans leur gratuité, leurs hasards, leurs combinaisons parfois saugrenues, tels qu'ils ont été : cette fidélité fait comprendre mieux que la plus adroite des transpositions comment les choses arrivent pour de bon aux hommes. Le danger, c'est qu'à travers cette profusion, le lecteur ne distingue aucune image claire, seulement un fatras. De même qu'il est impossible au physicien de définir à la fois la position d'un corpuscule et la longueur de l'onde qui lui est attachée, l'écrivain n'a pas le moyen de dire en même temps les faits d'une vie et son sens. Aucun de ces deux aspects de la réalité n'est plus vrai que l'autre ${ }^{29}$.

Les récits autobiographiques de Beauvoir ne cessent d'interroger les limites de cette représentation de la vie : l'incapacité de l'écriture rétrospective de soi à saisir la « poussière quotidienne de [1]a vie » conduit Beauvoir à intégrer à ses Mémoires des extraits de ses journaux d'époque ${ }^{30}$, et celleci souligne à de nombreuses reprises la difficulté qu'il y a à faire tenir dans des mots figés et définitifs le caractère changeant et ambigu de l'existence $^{31}$. L'autobiographie n'en est pas moins revendiquée comme la forme par laquelle la contingence de l'existence et son ambiguïté surgissent de la manière la plus fidèle à l'expérience humaine. L'œuvre à la première personne est donc philosophique en tant qu'elle est une forme de phénoménologie de la vie vécue.

Le cycle mémorial répond à un second niveau à la tension existentialiste entre démarche philosophique et rejet de l'universalisme philosophique en s'inscrivant dans la filiation rousseauiste de l'autobiographie comme témoignage non particulier mais singulier de ce qu'est une existence humaine. Il apparaît clairement dans les Mémoires que c'est d'abord comme lectrice que Beauvoir est impressionnée par la puissance de l'autobiographie : l'écriture du Deuxième Sexe est conçue comme le préalable

29. Id., La Force des choses I, dans Mémoires, t. II, op. cit., p. 219.

30. «J'ai tenu un journal, pendant cette période. En voici des extraits; ils livrent ce que ma mémoire échoue à ressusciter : la poussière quotidienne de ma vie. » (Id., La Force des choses I, op. cit., p. 1009-1010).

31. « À partir de 1943, mon bonheur avait été porté par les événements ; je collais si allégrement à mon temps que je n' avais rien à en dire. » (Ibid., p. 1205). « Un défaut des journaux intimes et des autobiographies c'est que, d'ordinaire, "ce qui va sans dire" n'est pas dit et qu'on manque l'essentiel. J'y tombe, moi aussi. Dans Les Mandarins, j' ai échoué à montrer combien le travail de mes héros comptait pour eux ; j'espérais ici mieux parler du mien : je me leurrais. Le travail ne se laisse guère écrire : on le fait, c'est tout. Du coup, il tient dans ce livre peu de place, alors que dans ma vie il en occupe tant : elle s'organise tout entière autour. [...] J'ai tenté de saisir la réalité dans sa diversité et sa fluidité ; résumer mon récit en mots définitifs, c'est aussi aberrant que de traduire en prose un bon poème. Le fond, tragique ou serein sur lequel mes expériences s'enlèvent leur donne leur vrai sens et en constitue l'unité ; j'ai évité de les lier par des transitions qui seraient univoques et donc artificielles. » (Ibid., p. 1215-1218). « J'ai relu ce journal et ça m'a amusée. Je devrais le continuer mais il faudrait le soigner davantage. Toujours «ce qui va de soi » est passé sous silence : par exemple nos réactions après l'exécution de Nagy. » (Id., La Force des choses II, op. cit., p. 151). 
d'une œuvre qui lui permettra de marcher dans le sillon de Michel Leiris ${ }^{32}$ et elle continuera jusqu'à la fin de sa vie à accorder une place de choix dans ses lectures à ce genre littéraire ${ }^{33}$. La référence aux Confessions de Rousseau traverse les Mémoires ${ }^{34}$ et met en évidence le risque de « flotter dans l'universe ${ }^{35}$ », inhérent à la philosophie. Beauvoir, lorsqu'elle justifie sa démarche, la présente comme une « enquête » dans laquelle sa vie apparaît comme la manifestation singulière d'une vie :

On me dira peut-être que ce souci ne concerne que moi ; mais non ; Samuel Pepys ou Jean-Jacques Rousseau, médiocre ou exceptionnel, si un individu s'expose avec sincérité, tout le monde, plus ou moins, se trouve mis en jeu. Impossible de faire la lumière sur sa vie sans éclairer, ici ou là, celle des autres. [...] L'étude d'un cas particulier renseigne mieux que des réponses abstraites et générales : c'est ce qui m'encourage à examiner le mien. Peut-être cet exposé aidera-t-il à dissiper certains des malentendus qui séparent toujours les auteurs de leur public et donc j' ai éprouvé bien souvent le désagrément ; un livre ne prend son vrai sens que si l'on sait dans quelle situation, dans quelle perspective et par qui il a été écrit : je voudrais expliquer les miens en parlant aux lecteurs de personne à personne.

Cependant, je dois les prévenir que je n'entends pas leur dire tout. [...] Des critiques ont cru que dans mes Mémoires j'avais voulu donner aux jeunes filles une leçon ; j'ai surtout souhaité m'acquitter d'une dette. Ce compte rendu en tout cas est dénué de toute préoccupation morale. Je me borne à témoigner de ce que ma vie a été. Je ne préjuge rien, sinon que toute vérité peut intéresser et servir ${ }^{36}$.

L'influence des premières pages des Confessions est ici très claire et manifeste la volonté de proposer par l'autobiographie l'analyse d'un cas particulier qui, sous l'effet du langage, devient non plus ce qu'il était, à savoir une vie parmi tant d'autres, mais une vie singulière, dans laquelle se reflète quelque chose de l'humanité tout entière. Écrire sa vie, ce n'est donc pas se proposer en modèle au sens de ce qui devrait être suivi, mais

32. «En fait, j' avais envie de parler de moi. J'aimais L'Âge d'homme de Leiris ; j'avais du goût pour les essais-martyrs où on s'explique sans prétexte. » (Id., La Force des choses I, op. cit. p. 1035).

33. «En général, les journaux intimes me fascinent, et celui-ci est assez extraordinaire, vraiment on plonge dans une autre vie, un autre système de référence et en un sens c'est la plus aiguë des contestations : pendant que je la lis, c'est elle le sujet absolu, ce n'est plus moi. » (Id., La Force des choses, dans Mémoires, t. II, op. cit., p. 130), « Je lus Les Lettres à Madame Z. de l'écrivain polonais Brandys ; et, en manuscrits, Derrière la baignoire de Colette Audry et Le Vieillissement de Gorz. Des œuvres très différentes, mais toutes les trois libres et directes ; elles me jetaient au cœur d'une expérience étrangère qui me reposait de moi, tout en me parlant de ce qui m'intéresse. » (Ibid., p. 332).

34. Voir par exemple La Force de l'âge, op. cit., p. 356 ; Tout compte fait, op. cit., p. 648 et 667. 35. « On m'aurait surprise et même irritée, à trente ans, si on m'avait dit que je m'occuperais des problèmes féminins, et que mon public le plus sérieux, ce serait des femmes. Je ne le regrette pas. Divisées, déchirées, désavantagées, pour elles plus que pour les hommes il existe des enjeux, des victoires, des défaites. Elles m'intéressent ; et j'aime mieux, à travers elles, avoir sur le monde une prise limitée, mais solide, que de flotter dans l'universel. » (Id., La Force 
en exemple au sens de ce qui montre une vérité possible de l'existence humaine. Ainsi que l'écrit Beauvoir, «par le langage je dépasse mon cas particulier, je communie avec toute l'humanité ${ }^{37} »$, c'est par l'écriture de la vie qu'apparaît une vérité plus large que celle de la vie de Beauvoir elle-même et qui permet de faire apparaître sa vie comme un « universel singulier ${ }^{38} \gg$.

Cette conception de l'écriture de soi comme manifestation du singulier contre l'universel et dans lequel seul le singulier permet de faire apparaître la tension entre liberté et situation est au cœur de l'entreprise philosophique beauvoirienne. Comme on l'a vu au sujet de son désaccord avec Sartre, Beauvoir reconnaît la place centrale de la liberté dans l'existence humaine mais insiste sur le fait qu'une telle liberté ne s'exprime qu'en fonction de la situation, politique, économique, historique, sociale, dans laquelle l'individu se trouve. Un tel accent sur le concept de situation est primordial car celui-ci va à l'encontre de l'individualisme propre à l'existentialisme : contre Sartre et avec Heidegger, Beauvoir souligne la préexistence du monde social et l'importance des normes sociales dans la liberté de l'individu. Faisant ainsi de sa propre vie un exemple singulier de la condition humaine, elle s'attache à travers toute son œuvre autobiographique à montrer à la fois la force de la situation qui est la sienne sur ses choix et sa liberté. Les Mémoires d'une jeune fille rangée montrent, comme le montre l'article indéfini dans le titre même, la tension entre une vie considérée comme suffisamment importante et singulière pour être racontée et l'ambition quasi sociologique du récit conçu comme le portrait de toute une classe sociale. Cette tension se retrouve dans le souci qu'a Beauvoir de mettre en lumière à la fois les éléments de son éducation typiquement bourgeoise et la façon dont sa liberté s'exprime. Mais également, en ce qui concerne La Force de l'âge, dans la critique des positions du couple durant l'entre-deux-guerres : «C'était contre condition de jeunes intellectuels petits-bourgeois qui nous incitait à nous croire inconditionnés ${ }^{39} »$. Rétrospectivement, Beauvoir fait apparaître la force du tournant philosophique qui sera le sien pendant la guerre. Celuici consiste, à nouveau contre Sartre, à reconnaître ce conditionnement par la situation et sera à l'origine aussi bien du Le Deuxième Sexe que de l'écriture des Mémoires. En effet, on peut faire l'hypothèse que le flou générique de l'œuvre à la première personne de Beauvoir est précisément la conséquence de ce qu'elle nomme dès 1946 l'ambiguïté de l'existence et qui tient dans cette tension entre liberté et situation. Comme l'écrit JeanLouis Jeannelle, la distinction entre l'autobiographie et les Mémoires tient en ce que la première « rend compte de ce qui distingue un sujet » quand 
les seconds « sont le récit d'une vie dans sa condition historique ${ }^{40}$ ». Or Beauvoir cherche précisément à montrer à la fois son « conditionnement ${ }^{41}$ " et sa liberté, qui, elle le reconnaît sans cesse, est plus difficile à exprimer. À ce titre, la dimension autobiographique de cette œuvre, le récit de ses états affectifs, de ses doutes, l'appréciation rétrospective de ses erreurs, est indissociable de la condition historique, sociologique, économique dans laquelle elle se trouve et que manifeste la dimension mémoriale de son œuvre. Seule l'union de l'autobiographie et des Mémoires peut espérer rendre compte à la fois de la liberté et de la situation qui sont les siennes et qui, ensemble, font son existence.

L'écriture de la vie, enfin, est philosophique chez Beauvoir en tant qu'elle est le seul moyen, quoique très imparfait, de faire surgir cet élément de la vie que la vie manque irrémédiablement, c'est-à-dire le présent. Les Mémoires sont tout entiers traversés par la conscience aiguë que Beauvoir a du temps qui passe et de la mort qui approche. Si l'influence de Bergson sur Beauvoir est drastiquement sous-évaluée dans Les Mémoires d'une jeune fille rangée par rapport à la place qu'elle occupe dans les Cahiers de jeunesse, la philosophe est manifestement très marquée par la question de la durée et du caractère insaisissable du temps qui s'écoule. Ainsi écritelle, dans les dernières pages de La Force des choses :

J'ai vécu tendue vers l'avenir et maintenant, je me récapitule, au passé : on dirait que le présent a été escamoté. J'ai pensé pendant des années que mon œuvre était devant moi, et voilà qu'elle est derrière : à aucun moment elle n'a eu lieu. Ça ressemble à ce qu'on appelle en mathématique une coupure, ce nombre qui n'a de place dans aucune des deux séries qu'il sépare ${ }^{42}$.

Ce passage, lorsqu'il est mis en parallèle avec la difficulté d'exprimer la liberté, au contraire de la situation ${ }^{43}$, fait apparaître la fonction autobiographique de l'œuvre de Beauvoir : il s'agit de saisir par l'écriture un présent ayant été vécu mais non saisi, en le faisant surgir comme un passé. Le propre du présent est d'être insaisissable car il est précisément, comme la coupure mathématique, la démarcation du passé et du futur. Or la liberté s'exprime dans le présent ; elle est la façon dont la situation est à chaque instant réappropriée par le sujet libre. L'ambition de l'œuvre à la première personne chez Beauvoir est précisément de montrer une façon singulière de reconnaître sa situation tout en la transcendant par sa liberté, et seule l'écriture, qui parvient à figer le présent dans l'épaisseur des mots, peut y répondre.

40. Jean-Louis Jeannelle, Écrire ses Mémoires aux XXe siècle, op. cit., p. 13.

41. Simone de Beauvoir, La Force des choses I, op. cit. p. 938-939.

LITTÉRATURE

42. Id., La Force des choses II, op. cit., p. 377. 
S'il ne peut être question d'épuiser une fois pour toutes la portée philosophique des milliers de pages qu'écrit Beauvoir sur sa vie, le fil existentialiste du lien entre la vie et la philosophie permet de montrer plusieurs choses. Premièrement, les Mémoires fournissent une source documentaire immense pour comprendre les essais de Beauvoir et la position philosophique originale que celle-ci y défend. Deuxièmement, ils permettent de mettre en évidence le lien à double sens entre la philosophie et la vie, sur le plan épistémique comme sur le plan pratique. Troisièmement, l'écriture de la vie permet de pallier les défauts inhérents à la philosophie lorsque celle-ci s'exprime en troisième personne : seul le témoignage littéraire permet de faire surgir un universel singulier qui témoigne par l'exemple de la tension entre situation et liberté et donne à voir un présent que son immédiateté rendrait, sans la littérature, irrémédiablement insaisissable. 